\title{
Hacia un abordaje funcional del volumen sanguíneo en la hemodinámica clínica
}

\section{Towards a functional approach to blood volume in clinical hemodynamics}

Rafael Dalmau ${ }^{1}$

análisis habitual del volumen sanguíneo como variable hemodinámica carece hoy día de un sentido integral. Debido a que el objetivo general del manejo hemodinámico consiste, al mismo tiempo, en preservar la homeostasis de la volemia y la perfusión tisular, estos dos aspectos de diferente naturaleza no son fácilmente "conciliables".

En esta Editorial, ahondaremos en esta cuestión, con vistas a plantear un abordaje realista e integral al volumen sanguíneo, y desde un punto de vista funcional.

I

Para comenzar, somos introducidos a los conceptos de "efectividad" y "estrés" cada vez que tratamos con la dinámica del volumen sanguíneo. El "volumen circulante efectivo"a es definido como el volumen alojado en el sistema o compartimiento arterial[1]; y, el "volumen sanguíneo estresado"b se refiere estric- tamente a la fracción de la volemia que distiende la vasculatura en su totalidad[2],[3], -es decir, que genera presión transmural, más allá de su volumen de repleción.

Si bien se trata de definiciones diferentes, las dos apuntan a lo mismo: la perfusión; una fracción de la volemia que "hace a la circulación", que perfunde los tejidos, que es "activa" hemodinámicamente. Mientras que la contrapartida de estas fracciones -el volumen "no-estresado", y "no-efectivo"- tienen una función, no completamente definida, de reservorio sanguíneo; hasta el extremo de llegar a suponer que aquel volumen no circula o que ambas fracciones coexisten y circulan independientemente[3],[4],[5].

Asimismo, estas interpretaciones esconden otra noción intuitiva, quizás más sutil: el flujo sanguíneo es relacionado causalmente con la presión del volumen sanguíneo en cuestión, -el volumen "presurizado" es el que circula[6]; la presión es de algún modo primaria al flujo[7],[8]. Para continuar aclarando el panorama,

Médico anestesiólogo. Servicio de Anestesiología, Hospital Español de Rosario, Rosario, Argentina.

Fecha de recepción: 17 de julio de 2018

Fecha de aceptación: 23 de julio de 2018

\section{ORCID}

https://orcid.org/0000-0002-8378-8221

Correspondencia:

Email: rdalmau@hotmail.com

a El concepto de volumen circulante efectivo es empleado clásicamente en fisiología renal y del medio interno. Se refiere al volumen arterial, en contraposición al resto del volumen intravascular e intersticial (compartimiento extracelular del agua corporal total)[1].

b Los términos volumen "estresado" y "no-estresado" han sido utilizados legítimamente en diversas áreas de la fisiología circulatoria, especialmente en modelos hemodinámicos computacionales. El aspecto de esta terminología que se critica aquí hace referencia a una peculiar malinterpretación que se ha instaurado en la literatura desde fines de la década de 1990[2], en el contexto del modelo Guytoniano del "retorno venoso" sistémico y los "análogos de la bañadera"[2],[3] (ver texto y referencias respectivas). 
a continuación explicaremos someramente el error en cada una de estas concepciones (para mayor detalle del análisis, consultar las referencias respectivas).

Volumen circulante efectivo. El volumen intra-vascular es (virtualmente) constante y el circuito cerrado; lo cual implica que: 1) el volumen alojado en el árbol arterial, en cierto momento, ha circulado por el sistema venoso y volverá a hacerlo nuevamente por el sistema arterial; con lo cual: 2) no tiene sentido alguno "priorizar" un compartimiento vascular (y su volumen sanguíneo contenido) ya que cada segmento es funcionalmente interdependiente del otro, por el solo hecho de que el flujo atraviesa la totalidad del circuito, y que: 3) es la tasa de flujo volumétrico la que determina el volumen de los sistemas arterial y venoso[9].

En síntesis, es el flujo, en todo caso, el que "efectiviza" al volumen sanguíneo; y la función del sistema venoso repercute necesariamente sobre la función arterial y viceversa.

Volumen sanguíneo estresado. Este concepto confronta directamente la física de la hidrostática. Establece que el volumen total de un compartimiento distensible (como el vascular) se compone, real y literalmente, de las dos fracciones mencionadas y con propiedades hemodinámicas diferentes, y se aplica generalmente al volumen venoso[3]-[6].

1) Esta noción está basada en una interpretación abstracta de la curva presión-volumen (la elastancia) de la vasculatura[8], en donde se identifican dos segmentos (relación no-lineal) y los dos volúmenes; pero estos volúmenes son virtuales y su separación simplemente no tiene correlato físico real: la presión en un sistema compliante y colapsable puede ser negativa, cero o positiva, pero siempre es homogénea; no se encuentra "fraccionada"[8],[10],[11].

2) Otra malinterpretación ligada a esta dicotomía entre volúmenes está relacionada con su localización en la vasculatura: se dice, que el volumen no-estresado se encuentra principalmente en los segmentos venosos periféricos[2]-[6], en donde se aloja la mayor parte de la volemia a presión baja, dado su gran capacitancia y tamaño. Pero este volumen está, por definición, "estresado" (es decir, su presión es baja, pero no cero) [10]; el único segmento del circuito en donde el volumen sanguíneo puede realmente llegar a encontrarse "no-estresado" es en los segmentos corriente-abajo -el compartimiento venoso central[11] -en donde la presión sanguínea se aproxima a cero, dependiendo de varios factores. La implicancia funcional y práctica de la presión venosa central cayendo a cero, estriba en que el colapso de los vasos a este nivel opondrá resistencia al flujo comprometiendo la precarga ventricular derecha y por consiguiente, el gasto cardíaco[11],[12].

3) Es interesante notar que esta noción "encaja" en el modelo del "retorno venoso sistémico" de Guyton", en el cual la presión media de lleno circulatorio es entendida como la presión generada por la fracción "estresada" de la volemia, allí en el compartimiento venoso periférico, responsable del flujo de retorno venoso[2]-[6]; cuando esta presión es simplemente el cociente entre el volumen sanguíneo total y la compliancia del sistema[7]-[11], y solo eso.

4) Por último, el concepto de "estrés" aplicado al volumen sanguíneo es redundante, ya que mienta lo mismo que la presión (hidrostática) sanguínea; a diferencia del estrés parietal de los vasos sanguíneos y del músculo cardíaco, que es una medida de la carga mecánica que experimentan estas estructuras (estrés "normal"), y del estrés de cizallamiento que se genera en la sangre al fluir, producto de su viscosidad (estrés "tangencial" o "cortante").

Así, una noción abstracta y últimamente errónea de "efectividad" del volumen sanguíneo es creada a través de estas concepciones; la diferencia es, si se quiere, "anatómica": el concepto de "volumen circulante efectivo" se aplica al sistema arterial y el "volumen estresado" al venoso. En las siguientes secciones esta idea de efectividad será reinterpretada.

\section{II}

En la sección anterior, hemos comenzado por descubrirc el volumen sanguíneo para continuar ahora analizando esta variable, ya sin abstracciones. Una reseña sobre su funcionalidad, reología e histología excede, lógicamente, el alcance de este ensayo. Ahora, nos enfocaremos en el aspecto hemodinámico del volumen sanguíneo en su totalidad (esto es, como variable real), y en relación al mantenimiento de su homeostasis como tejido.

Vale decir que este aspecto ha sido conceptualmente descartado en la hemodinámica clínica moderna, especialmente, la aplicada a la medicina intensiva y perioperatoria; lo cual es contradictorio, ya que cualquier desvío cualicuantitativo de la normalidad del volumen sanguíneo (por ejemplo, hemorragias, deshidratación, "tercer espacio", sobrecarga, anemia, etc.) alertan, lógicamente, al especialista, y lo "hacen

c En el sentido literal etimológico de "descubrir" (discooperire): "quitar aquello que cubre o tapa algo que antes había permanecido oculto". 
pensar" inmediatamente en esta variable tal como es, y no en las "fracciones" mencionadas en la sección anterior. Asimismo, llevar un "balance hídrico" es una práctica no solo en desuso, sino hasta temáticamente desacreditada por las tendencias modernas en fluidoterapia.

Por un lado, como ya vimos, se advierte que "no importa el volumen sanguíneo total, sino la 'fracción activa' del mismo ('estresada' y/o 'efectiva')"[12],[13]. $Y$, por otro lado, también se dice que aquello que es relevante respecto al conocimiento sobre el estado volémico no es, nuevamente, la totalidad de la volemia, sino "la capacidad de respuesta a fluidos" del paciente[14]. Este último argumento forma parte de otra metodología en hemodinámica clínica que se ha consolidado desde fines de la década de 1990, hasta hoy: la hemodinámica funcional y el concepto de la "respuesta a fluidos"d.

Si bien se trata de aproximaciones diferentes al estado volémico ("volumen estresado/efectivo", y "respuesta a fluidos"), ambas convergen en el tratamiento que se hace del volumen sanguíneo; esto es, la fluido-terapia es guiada por variables (cuya interpretación, como hemos visto, es cuestionable) que no tienen que ver con la homeostasis de la volemia. La misma es conceptualmente descartada como variable, cuando, en la realidad, toda fluido y hemoterapia recaen sobre el volumen sanguíneo, el cual es desconocido[14].

Información fundamental sobre esta variable, como el volumen sanguíneo total, volumen globular, volumen plasmático y hematocrito normalizado son tenidas en cuenta en especialidades médicas que tratan rutinariamente con el volumen sanguíneo[15]-[17]. Es decir, la volemia no solo es una variable medible en la práctica clínica, sino también analizable[15].

Un avance consiste en comprender que el volumen sanguíneo es "efectivo" en sí mismo y en su totalidad; en el sentido de que es toda la volemia la que tiene funciones y efectos, y que excesos y déficits de la misma son demostrablemente deletéreos[18]. ¿Cómo es entonces que, en la era de la "fluido-terapia guiada por objetivos", la volemia no es un objetivo en sí mismo?

III

¿Qué ocurre, entonces, con el volumen sanguíneo? ¿Cómo podemos hablar de su dinámica, y de su "efectividad" hemodinámica?

Todos sabemos que la función hemodinámica de la sangre pasa por la perfusión; es decir, su fluir. Una volemia "normal", como fue presentada en la sección anterior, no cumple su función si no fluye adecuadamente. La clásica ecuación para la entrega sistémica de oxígeno $\left(\mathrm{DO}_{2}\right)$ ilustra fácilmente la dependencia de la $\mathrm{DO}_{2}$ sobre el transporte de oxígeno en sangre (presión parcial del gas, concentración y saturación de la hemoglobina) y el gasto cardíaco[13]. El manejo hemodinámico, entonces, debe lograr el equilibrio entre la optimización de ambas funciones -oxigenación y flujo- cuidando que no interfieran entre sí (por ejemplo, una fluido-terapia excesiva dirigida a aumentar el flujo que, al diluir la sangre, disminuya la oxigenación).

El gasto cardíaco, a su vez, depende de las propiedades físicas y fisiológicas del corazón y de la vasculatura, y de la interacción entre ambos subsistemas[9],[10],[19]. Siendo la bomba cardíaca la fuente de energía mecánica para el flujo estacionario[7]-[9],[19],[20], la terapéutica debiera enfocarse a optimizar la función cardíaca. Pero, ni las propiedades intrínsecas del músculo cardíaco (como el mecanismo de Frank-Starling) ni la regulación neurohumoral extrínseca del corazón pueden sobreponerse a lo que la vasculatura falla en proveer como precarga[21]; es decir, que la precarga -principal determinante del volumen sistólico- no es un fenómeno "aislado"; depende del acoplamiento entre la bomba y el circuito vascular a nivel derecho o de "baja presión" ("acoplamiento veno-ventricular"[8],[22]), y más importante aún, este acoplamiento es crítico en aquellas situaciones en donde el tono vaso-motor puede ser abruptamente alterado, como en la anestesiología[23].

¿En qué consiste, entonces, esa tan buscada "intersección" entre el volumen sanguíneo y la circulación? ¿Qué es aquello del volumen sanguíneo que afecta su circulación, y que lo hace, si se quiere, "efectivo"? Es su distribución.

Este concepto -la distribución del volumen sanguíneo- quizás no resulte tan familiar como lo es la "distribución del flujo". Ello se debe, en parte, a la falta de énfasis sobre un "ingrediente" olvidado y malinterpretado en hemodinámica: la compliancia vascular[8]. Si la vasculatura fuese rígida, el flujo sanguíneo (y su distribución) no afectarían la precarga ventricular; el gasto cardíaco sería únicamente gobernado por la contractilidad, frecuencia y poscarga cardíacas. Pero la perfusión tiene, por así decirlo, un "costo": la acumulación periférica de volumen sanguíneo.

d Consultar Revisión del mismo autor en el presente Número; un análisis crítico de la rationale de la "respuesta a fluidos"[14]. 
El volumen de un segmento o compartimiento vascular periférico depende de su compliancia y de su perfusión, y dado que la volemia es constante, el volumen (y presión) vascular periférico guardan una relación inversa con el central (precarga)[8],[19]. Así, esta relación inversa y proporcional entre la precarga y el gasto cardíaco (para el circuito en su totalidad) conforma un mecanismo hidráulico pasivo para la regulación del flujo sistémico[19]-[24].

Es importante, entonces, conocer cómo los diversos patrones de distribución del volumen sanguíneo afectan la precarga ventricular y, por lo tanto, el gasto cardíaco. Para ello, podemos describir tres instancias de distribución:

1) Distribución: distribución cuando el flujo es cero. Luego del paro circulatorio, el perfil de presiones vasculares se equilibra y la presión sanguínea en todo el circuito es homogénea (entre 10 y $20 \mathrm{~mm} \mathrm{Hg}$ ). Pero, dado que la compliancia vascular es heterogénea (el sistema venoso es veinte veces más compliante que el arterial, aproximadamente) el perfil de volemia también lo es: más volumen en las venas y menos en las arterias[9],[19],[22] la volemia se distribuye.

2) Redistribución: cuando hay flujo (estacionario), parte del volumen venoso es bombeado al sistema arterial, cambiando el perfil de presión acorde (la presión arterial aumentará 20 veces más de lo que la presión venosa central disminuye por cada latido), en proporción a la tasa de flujo[9],[22], distribución flujo-dependiente[19]. Aquí, debemos destacar dos tipos de redistribución; dado que la circulación periférica se compone de lechos de alta y baja compliancia, la acumulación de volumen sanguíneo en la periferia dependerá de la proporción del tipo de lecho perfundido o distribución intercompartimental.

3) Mal-distribución[27]: cualquier situación en la que el volumen vascular central sea insuficiente para permitir una precarga y gasto cardíaco adecuados para suplir necesidades tanto metabólicas (consumo tisular de oxígeno) como funcionales (perfusión cutánea para disipación de calor corporal). Por lo tanto, este escenario cursará con presiones vasculares centrales bajas y periféricas altas.

También, hay que remarcar que no todos los casos de "maldistribución" serán de alto flujo y metabolismo. En la ortostasis (Figura 1), por ejemplo, 80\% del volumen sanguíneo se encuentra por debajo del nivel del corazón y, por ello, los volúmenes y gasto cardíacos son bajos; la distribución periférica del volumen sanguíneo no es en este caso flujo-dependiente, sino que depende de la distensión hidrostática de los segmentos venosos caudales. Similarmente, la vasodilatación pasiva o activa producirá el mismo patrón de distribución por la misma causa.

Dado que las presiones y volúmenes vasculares periféricos no son accesibles a la medición clínica cotidiana, cualquier caso de maldistribución debe ser distinguido de la hipovolemia (en su fase de descompensaciónf), en donde los marcadores de precarga serán también bajos. Más aún, la hipovolemia descompensada y la maldistribución (que puede cursar, idealmente, con hipo, normo, o hipervolemia) son indistinguibles si no contamos con 1) la historia clínica; 2) medición del volumen sanguíneo y/o del gasto cardíaco, ya que en ambas situaciones las presiones y volúmenes centrales serán bajas.

Es así, como la normovolemia adopta una definición "dual" o "combinada"; por un lado, podemos definirla en términos absolutos -como cualquier otra variable hemodinámica-, según variables antropométricas, y por otro lado, según su patrón de distribución, es decir, en relación a la precarga ventricular.

Como conclusión, el autor sugiere que el abordaje clínico al volumen sanguíneo deba contemplar integralmente tres aspectos (interrelacionados) de esta variable:

1) Su homeostasis: volumen sanguíneo total, globular, plasmático y hematocrito normalizado ${ }^{15}$.

2) Su distribución: en relación al volumen vascular central25,26.

3) Su funcionalidad: volumen minuto cardíaco y transporte de oxígeno[13].

e La distinción entre las "tres instancias de distribución" es meramente formal. Para estudiar en detalle las posibilidades de distribución del volumen sanguíneo y su relación con el gasto cardíaco, precarga, y consumo de oxígeno en la circulación humana intacta, consultar los trabajos experimentales al respecto de Rowell LB y colaboradores[25],[26].

f Se define a la fase de descompensación de la hipovolemia, cuando el ajuste, pasivo y activo, de la capacitancia vascular periférica ya no logra mantener una precarga ventricular adecuada[28] y, por lo tanto, la hipovolemia se torna sintomática, con presiones de lleno bajas, gasto cardíaco bajo, taquicardia e hipotensión arterial. 


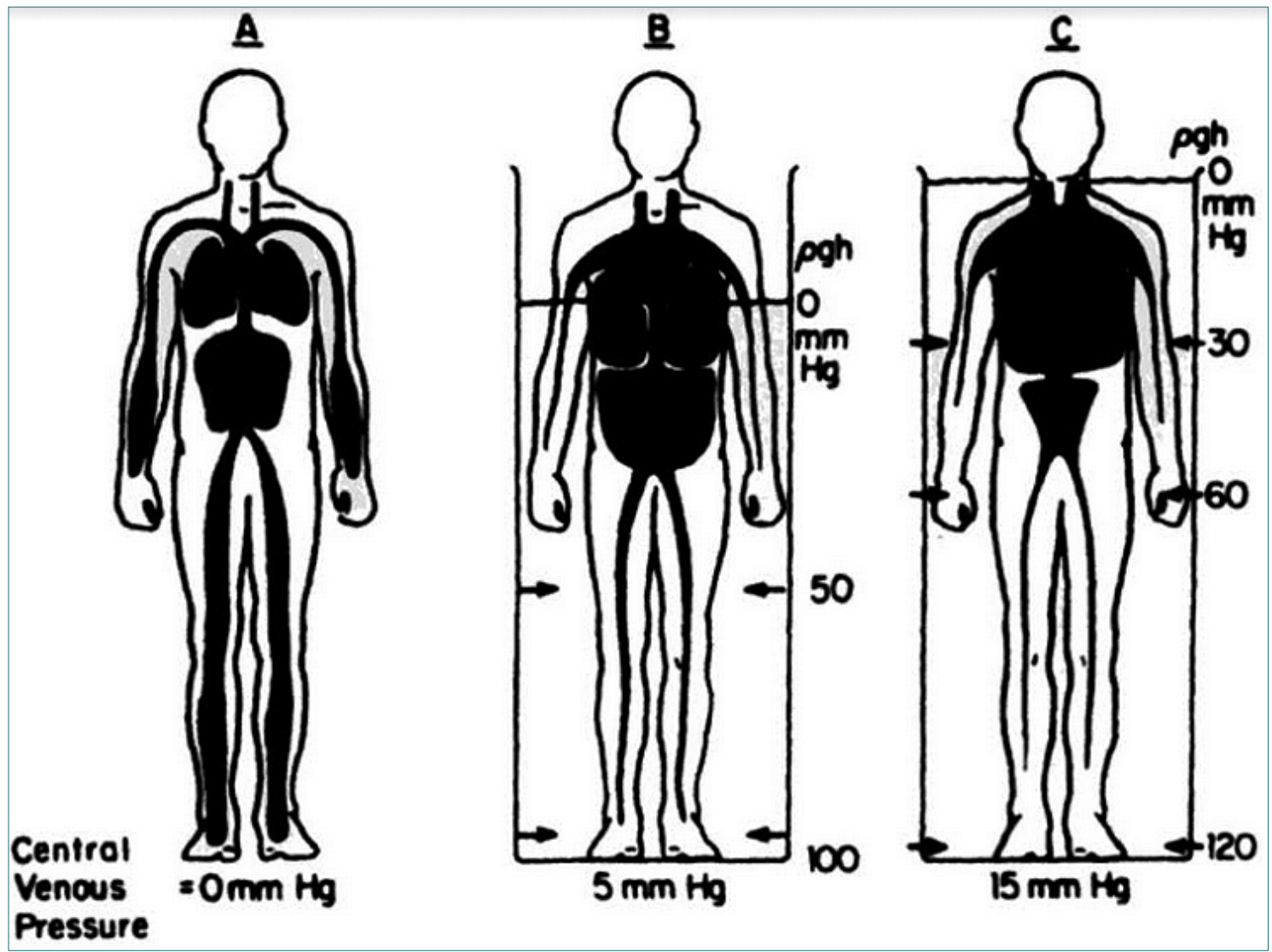

Figura 1. Distribución del volumen sanguíneo en la ortostasis y la inmersión en agua termoneutra. En el panel " $A$ " aproximadamente el $80 \%$ de la volemia se encuentra distribuida por debajo del nivel del corazón y, por lo tanto el volumen y presión del compartimiento venoso central intratorácico son bajos (presión venosa central de 0 mm Hg); la volemia en el bípedo se encuentra normalmente "maldistribuida" en la ortostasis. Panel "B": la inmersión en agua hasta el nivel del apéndice xifoides transloca pasivamente parte de la volemia hacia el tórax elevando el volumen y presión venosa central (5 mm Hg). Panel "C": la inmersión progresa hasta la base del cráneo, causando ahora la ingurgitación de los vasos intratorácicos, a una presión de $15 \mathrm{~mm} \mathrm{Hg}$. Nótese que el volumen sanguíneo es constante, y que los marcadores de precarga cambiaron radicalmente como consecuencia del cambio en el patrón de la distribución del volumen sanguíneo (de la periferia hacia el centro); el gasto cardíaco también se asume invariable (de reposo), si bien el aumento progresivo de la precarga reclutará el mecanismo de Frank-Starling, aumentando el volumen sistólico. "Central Venous Pressure": Presión Venosa Central; mm Hg: milímetros de mercurio; $\rho$ : densidad; g: aceleración gravitacional; h: altura ( $\rho g h$ : presión hidrostática). Extraído de: Rowell LB. Passive Effects Of Gravity. En: Human Cardiovascular Control. New York. Oxford Univ. Press, 1993, pp. 23.

\section{Referencias}

1. Schrier RW. An odyssey into the milieu intérieur: pondering the enigmas. J Am Soc Nephrol. 1992 May;2(11):1549-59.

2. Magder S, De Varennes B. Clinical death and the measurement of stressed vascular volume. Crit
Care Med. 1998 Jun;26(6):10614.

3. Gelman S. Venous function and central venous pressure: a physiologic story. Anesthesiology. 2008 Apr;108(4):735-48.

4. Gelman S, Bigatello L. The physiologic basis for goal-directed hemodynamic and fluid therapy: the pivotal role of the venous circulation. Can J Anaesth. 2018 Mar;65(3):294-308.

5. Magder S. Volume and its relationship to cardiac output and venous return. Crit Care. 2016 Sep;20(1):271.

6. Siegenthaler N, Giraud R, Bendjelid K. A semantic point of 
view about unstressed blood volume. Crit Care Med. 2013 Sep;41(9):e237-9.

7. Brengelmann GL. Counterpoint: the classical Guyton view that mean systemic pressure, right atrial pressure, and venous resistance govern venous return is not correct. J Appl Physiol (1985). 2006 Nov;101(5):15256.

8. Dalmau R. Hemodinámica: the Big Picture. Un análisis y modelo integrador de la circulación humana relevante para la hemodinámica clínica. Rev Argent Anestesiol. 2017;75(3):85-92.

9. Levy MN. The cardiac and vascular factors that determine systemic blood flow. Circ Res. 1979 Jun;44(6):739-47.

10. Dalmau R. The "stressed blood volume" revisited. Can J Anaesth. 2018 Jun; 7:

11. Brengelmann GL. Stressed and unstressed volumes of the peripheral vasculature. Can J Anaesth. 2018 Jun;1-2:

12. Repessé $X$, Vieillard-Baron A. Reply to "Letter to the editor: Comments on 'Value and determinants of the mean systemic filling pressure in critically ill patients'". Am J Physiol Heart Circ Physiol. 2015; 309(8): H1372-3.

13. Magder S. Flow-directed vs. goal-directed strategy for management of hemodynamics. Curr Opin Crit Care. 2016 Jun;22(3):267-73.

14. Dalmau R. Hemodinámica
Funcional: ¿Qué significa "ser respondedor"? Rev Chil Anest; 2018; 47:176-188.

15. Manzone TA, Dam HQ, Soltis D, Sagar VV. Blood volume analysis: a new technique and new clinical interest reinvigorate a classic study. J Nucl Med Technol. 2007 Jun;35(2):55-63.

16. Manzone H, Dam H, HowardSachs J. "Normalized hematocrit" (nHct) from blood volume analysis (BVA) offers enhanced accuracy over peripheral hematocrit (pHct) in assessment of red blood cell volume J Nucl Med May. 2010; 51: 1676.

17. Takanishi DM, Yu M, Lurie F, Biuk-Aghai E, Yamauchi $H$, Ho HC, et al. Peripheral blood hematocrit in critically ill surgical patients: an imprecise surrogate of true red blood cell volume. Anesth Analg. 2008 Jun;106(6):1808-12.

18. Peng ZY, Kellum JA. Perioperative fluids: a clear road ahead? Curr Opin Crit Care. 2013 Aug;19(4):353-8.

19. Brengelmann GL. A critical analysis of the view that right atrial pressure determines venous return. J Appl Physiol (1985). 2003 Mar;94(3):849-59.

20. Galetti VM. An analytical perspective on the venous return controversy. Can J Anaesth. 2018 Jun; 1 :

21. Rowell LB. General Principles of Vascular Control. Human Circulation. Regulation During Phy- sical Stress. New York: Oxford

Univ. Press; 1986. p. 9.

22. Tyberg JV. How changes in venous capacitance modulate cardiac output. Pflugers Arch. 2002 Oct;445(1):10-7.

23. Gelman S. Is norepinephrine infusion during intraoperative period justified? Anesthesiology. 2014 Aug;121(2):433-4.

24. Zidon TM, Sheriff DD. Diversion of blood flow from noncompliant to compliant vasculature in awake dogs: mechanical impact on right atrial pressure. Am J Physiol Heart Circ Physiol. 2006 Jan;290(1):H217-23.

25. Rowell LB, Murray JA, Brengelmann GL, Kraning KK 2nd. Human cardiovascular adjustments to rapid changes in skin temperature during exercise. Circ Res. 1969 May;24(5):711-24.

26. Rowell LB, Brengelmann GL, Blackmon JR, Murray JA. Redistribution of blood flow during sustained high skin temperature in resting man. J Appl Physiol. 1970 Apr;28(4):415-20.

27. Brengelmann GL. Letter to the editor: Comments on "Value and determinants of the mean systemic filling pressure in critically ill patients". Am J Physiol Heart Circ Physiol. 2015; 309(8): H1370-1.

28. Shen T, Baker K. Venous return and clinical hemodynamics: how the body works during acute hemorrhage. Adv Physiol Educ. 2015 Dec;39(4):267-71. 